\title{
Interactive software: an educational/behavioural approach to smoking cessation for pregnant women and their families
}

\author{
Walter J Scott, Helen McIlvain
}

Smoking cessation programs that have been used during pregnancy include brochures, physician advice, and counselling. Standardised self help brochures alone have minimal impact ${ }^{1}$; tailored messages are more effective. ${ }^{2}$ Face to face counselling requires training and is time consuming in a busy clinic. Multimedia (non-text based) approaches can reach populations with low literacy levels. Interactive digital media also allows users to learn by making choices and then noting the consequences of their actions. Health messages can be tailored to the user in real time. We designed a digital, interactive smoking cessation intervention tailored for pregnant women and tested it in public health clinics.

\section{Methods}

The study population consisted of pregnant smokers enrolled in the Douglas County (Omaha), Nebraska Women, Infants and Children (WIC) program. The mission of the WIC program is to decrease the number of low birth weight babies born to low income or otherwise at risk women. At the time the study was initiated, the most recent 12 month data available from the Centers for Disease Control (CDC) Pregnancy Nutrition Surveillance program was from 1992 . These data indicated that $31.9 \%$ of Douglas County WIC participants smoked during pregnancy. Pregnant women who were current smokers (defined as a client who "self reported smoking at least one puff of a cigarette in the last seven days") were eligible for study participation. Informed consent was obtained following guidelines of the Creighton University institutional review board. Women received vouchers from WIC on a monthly basis and this provided an opportunity for us to collect data. Incentives ( $\$ 10$ vouchers for merchandise at a local store-marked "not for alcohol or tobacco") were given to participants at the completion of each study visit.

The study design was sequential. The first component was a natural history study during which pregnant smokers were enrolled and followed to determine their rate of smoking cessation in response to the usual care delivered in the WIC clinics. These women received a brief intervention at the time of study enrollment. Next, a second group of pregnant smokers was enrolled in a pilot study of the intervention. On the second and third clinic visits, these participants used an interactive digital video and received a printout with tailored information to take home. At the first and fourth monthly visit to the clinic following study enrollment, saliva samples were taken from both groups and sent to a central laboratory for determination of salivary cotinine.

The primary end point of the study was the difference in the biochemically validated quit rates between the two groups of participants (the comparison group and the intervention group) at the end of pregnancy (fourth clinic visit). Secondary end points included differences by group in the mean number of reported cigarettes smoked at the end of pregnancy and in the number of women who reported advancing in their stage of readiness for change at the end of pregnancy compared to the beginning of the study.

The digital video intervention placed the user in the role of helper or advisor to the pregnant women who were presented in the video segments. For example, a pregnant woman in one of the videos asked, "My doctor told me that I was pregnant! He also said that I should quit smoking because smoking could hurt my baby. I don't get it, how can my smoking hurt my baby?". The user is presented with several options. She could immediately choose a response to give to the woman in the video, or she could first ask an expert for more information or advice, or she could ask another pregnant woman for advice. Before being allowed to move onto the next video segment, the user had to eventually give the correct advice to the pregnant woman asking the question in the video. Principles of motivational interviewing ${ }^{3}$ were followed in writing the scripts for the interactive videos. The intervention was tailored for the stage of change of the user. ${ }^{4}$

Based on her response to questions presented at the beginning of the interactive video, a woman was classified as a precontemplator, a contemplator, or as being in the preparation/action stage. If she answered "No" to the question "Are you thinking of quitting smoking in the next six months?", she was considered a precontemplator. If she answered "Yes" to that question, and then "No" to the next question "Are you thinking of quitting smoking in the next 30 days?", she was classified as a contemplator. If she answered 
Table 1 Baseline characteristics of comparison and intervention groups *

\begin{tabular}{lll}
\hline Characteristic & $\begin{array}{l}\text { Comparison group } \\
(n=119)\end{array}$ & $\begin{array}{l}\text { Intervention group } \\
(n=114)\end{array}$ \\
\hline Mean (SD) age (years) & $23.2(7.3)$ & $24.6(5.7)$ \\
Mean (SD) weeks pregnant & $13.9(6.6)$ & $14.9(6.6)$ \\
Mean (SD) cigarettes/day in previous 7 days & $9.8(8.3)$ & $10.0(13.4)$ \\
Education (\% completing grade 12) & $70.1 \%$ & $77.2 \%$ \\
Race (\%white) & $73.7 \%$ & $82.2 \%$ \\
\hline
\end{tabular}

^ No significant differences were found between groups at baseline.

"Yes" to both questions, she was considered to be in the preparation/action stage. An interactive digital video was developed for each of the three stages. At the end of each session, a printout was given to the women containing reinforcing messages tailored to their stage of change.

The hardware consisted of an interactive video player based on CD-ROM format with a touch screen monitor. Users activated and interacted with the intervention by touching the monitor screen. Text was highlighted as a narrator read it. A printer attached to the system provided a tailored message in real time.

Data were collected, stored, and analysed using SAS (Release 6.11, SAS Institute, North Carolina). Means and standard deviations were used to describe normally distributed data and t tests were used to compare these groups. Frequency data were compared between groups using $\chi^{2}$ tests or Fisher's exact test depending on the distribution of the expected values of each cell. A salivary cotinine concentration greater that $30 \mathrm{ng} / \mathrm{ml}$ was considered positive for current smoking.

\section{Results}

We recruited a total of 233 women into both parts of the study. Of those enrolled, 154 completed all parts of the study (one of the WIC clinics had a drop out rate of $70 \%)$. Drop out rates in the other two clinics were $17 \%$ and $20 \%$, a more acceptable rate for this patient population. The two groups were similar in terms of a number of important characteristics (table 1.) Of the 119 women in the comparison group, 75 finished the study (had complete data through the end-of-pregnancy measurement). Three of the 75 (4\%) women were biochemically confirmed quitters. Of the 114 women in the intervention group, 79 completed the study. Eight of the 79 were biochemically confirmed quitters, for a quit rate of $10.1 \%(p=0.211)$. If we assume that all of those lost to follow up are still smoking, then the quit rates become $3 / 119$ or $2.5 \%$ (comparison group) and $8 / 114$ or $7 \%$ (intervention group) $(\mathrm{p}=0.129)$.

The other major finding of this study concerned the stage of change of the participants in the two groups at the beginning and at the end of the study. At the time of enrollment, $24 / 118(20.3 \%)$ of those in the comparison group were precontemplators versus $32 / 114(28.1 \%)$ of those in the intervention group $(\mathrm{p}=0.169)$. By the end of pregnancy, $58 / 80(72.5 \%)$ of the comparison group were precontemplators as compared to $26 / 86(30 \%)$ of the intervention group $(\mathrm{p}=0.01)$.

\section{Discussion}

This study found a very low biochemically validated quit rate following usual care among WIC participants enrolled early in their pregnancies. We enrolled women who were pregnant for two or three months. Many women may have already quit smoking when they found out they were pregnant. The biochemically validated quit rate increased after the use of an interactive digital video intervention, but this was not statistically significant. If even a small improvement could be shown, this could have a significant public health impact, since the intervention could be made widely and cheaply available over the internet to kiosks in clinics and doctor's offices.

Of interest is the stage of change data in women as they progress through pregnancy. The percentage of precontemplators in the comparison group increased significantly by the end of pregnancy. If they were considering quitting but had not quit by the end of pregnancy, many women seemed to revert to precontemplation. This is consistent with clinical patterns since many women change their view of smoking as they reach the end of pregnancy (women who do quit during pregnancy often relapse in the immediate postpartum period). This large increase in precontemplators did not occur in the intervention group. If confirmed in larger studies, our intervention could help pregnant smokers eventually quit by preventing regression to an earlier stage of change by the end of pregnancy.

Supported by a grant from the Robert Wood Johnson Foundation, Smoke-Free Families Program.

1 Dijkstra A, DeVries H, Roijackers J, vanBreukelen G. Tailoring information to enhance quitting in smokers with low motivation to quit: three basic efficacy questions. low motivation to quit: three

2 Dijkstra A, DeVries H, Roijackers J. Targeting smokers with low readiness to change with tailored and nontailored selfhelp materials. Prev Med 1999;28:203-11.

3 Miller WR, Rollnick S. Motivational interviewing: preparing people to change addictive behavior. New York: Guilford Press, 1991.

4 DiClemente CC, Prochaska JO, Fairhurst SK, Velicer WF, Velasquez MM, Rossi JS. The process of smoking cessation: an analysis of precontemplation, contemplation, and preparation stages of change. $\mathcal{F}$ Consult Clin Psychol 1991;59:295-304. 